\title{
Tratamiento laparoscópico en atrapamiento del nervio ilioinguinal como causa de dolor agudo incapacitante: Reporte de caso y revisión de la literatura
}

\author{
Laparoscopic treatment in ilioinguinal nerve entrapment as a cause \\ of disabling acute pain: Case report and literature review

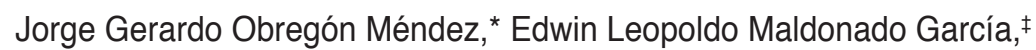 \\ César Óscar Decanini Terán, $\$$ Martin Vega de Jesús, ${ }^{\star}$ Iván López Yerena ${ }^{\ddagger}$ \\ * Alta Especialidad Cirugía Endoscópica, Centro Médico ABC. \\ ₹ Curso Posgrado de Alta Especialidad Cirugía Endoscópica, Centro Médico ABC. \\ \& Centro Médico ABC.
}

RESUMEN

Introducción: El dolor inguinal agudo se considera desde el posoperatorio inmediato hasta los 30 días. El dolor inguinal de origen neural es una complicación especialmente grave después de una hernioplastia. La lesión nerviosa puede ocurrir por traumatismo directo o por atrapamiento durante el proceso de cicatrización. Presentación del caso: Mujer de 42 años sin antecedentes patológicos de importancia; refiere antecedente quirúrgico de plastia inguinal bilateral laparoscópica hace tres semanas. Inició padecimiento actual en el posoperatorio inmediato hace dos semanas con dolor agudo en región inguinal izquierda tratada con neuromoduladores, ansiolíticos, antidepresivos y bloqueo neural por mapeo. Se realiza laparoscopía con tres puertos y búsqueda intencionada de la emergencia del nervio ilioinguinal a nivel del triángulo del dolor por laparoscopía, encontrando una grapa justo en la emergencia de dicho nervio; se hace retiro de malla previa debido a que presentaba migración y no cubría orificio inguinal profundo con colocación de nueva malla y fijación con pegamento, la paciente evoluciona de manera satisfactoria. Conclusión: El dolor agudo en el posoperatorio inmediato es una entidad que pocas veces requiere de exploración quirúrgica a menos que sea incapacitante y que no responda a las diferentes líneas de manejo conservador.

Palabras clave: Hernioplastia laparoscópica, nervio ilioinguinal, hernia inguinal.

\section{ABSTRACT}

Introduction: Acute inguinal pain is considered from the immediate postoperative period until 30 days. Inguinal pain of neural origin is an especially serious complication after a hernioplasty. Nerve injury can occur by direct trauma or by entrapment during the healing process. Case presentation: A 42-year-old female with no significant pathological history reported a surgical history of laparoscopic bilateral inguinal plasty three weeks ago. He began current condition in the immediate postoperative period two weeks ago with pain in the left inguinal region treated with neuromodulators, anxiolytics, antidepressants and neural block by mapping, laparoscopy is performed with three ports, intentional search for the emergence of the ilioinguinal nerve at the triangle level is performed of laparoscopic pain; finding a tack just in the emergency of said nerve and removal of previous mesh because it presented migration and did not cover deep inguinal hole with new mesh placement and fixation with glue, the patient evolved satisfactorily. Conclusion: Acute pain in the immediate postoperative period is an entity that rarely requires surgical exploration unless it is disabling and does not respond to the different lines of conservative management.

Keywords: Laparoscopic hernioplasty, ilioinguinal nerve, inguinal hernia.

\section{Recibido: 09/01/2020. Aceptado: 13/08/2020. \\ Correspondencia: Dr. Edwin Leopoldo Maldonado García \\ E-mail: edwinlmg@gmail.com}

Citar como: Obregón MJG, Maldonado GEL, Decanini TCÓ, Vega de Jesús M, López YI. Tratamiento laparoscópico en atrapamiento del nervio ilioinguinal como causa de dolor agudo incapacitante: Reporte de caso y revisión de la literatura. Rev Mex Cir Endoscop. 2020; 21 (3): 158-162. https://dx.doi.org/10.35366/99841 


\section{INTRODUCCIÓN}

El dolor inguinal agudo se considera desde el posoperatorio inmediato hasta los 30 días. Prácticamente, todos los pacientes refieren algún grado de dolor; sin embargo, es de fácil control mediante analgésicos y antiinflamatorios en todos los centros hospitalarios. ${ }^{1}$ Debe diferenciarse la neuralgia crónica del típico dolor agudo posquirúrgico; éste sucede los primeros días después de la cirugía, generalmente con mayor intensidad hasta el tercer día, además la intensidad del dolor suele estar relacionada con la extensión de la intervención y cede con analgésicos habituales. ${ }^{2}$ Según la Asociación Internacional para el estudio del dolor, la incidencia de dolor crónico (tres meses o más) después de una reparación inguinal con técnica transabdominal preperitoneal se presenta en $13.8 \%$ de los casos. ${ }^{3}$

El dolor inguinal de origen neural es una complicación especialmente grave después de hernioplastia. La lesión nerviosa puede ocurrir por traumatismo directo o por atrapamiento durante el proceso de cicatrización. Las manifestaciones clínicas de forma temprana son la presencia de dolor quemante y/o ardor sobre el área inguinal que se irradia al pubis, escroto o parte superior del muslo con percepción sensorial alterada sobre el dermatoma del nervio afectado. ${ }^{4}$

Los daños en los nervios inguinales se pueden producir de forma intraoperatoria o posoperatoria. En forma intraoperatoria se reconocen mecanismos como la manipulación quirúrgica, sobredisección, estiramiento, daño eléctrico o térmico, aplastamiento parcial o transección completa, fijación y/o atrapamiento en la sutura o medios de fijación pueden dañar los nervios. Los nervios susceptibles y los mecanismos de lesión variarán según el tipo de reparación y ubicación del nervio y la malla. Los abordajes exponen indirectamente a los nervios inguinales que pueden lesionarse dentro del canal inguinal con el uso de la fijación penetrante con dispositivos de fijación y suturas. ${ }^{5}$ La cirugía de mínimo acceso expone estructuras nerviosas encontradas dentro del «triángulo del dolor». En el espacio preperitoneal lateral, los nervios pueden lesionarse debido a la cicatrización perineural, irritación por fibrosis, atrapamiento, inflamación, infección, recurrencia, o formación de granuloma y/o neuroma. ${ }^{6}$

La anatomía del nervio ilioinguinal, al igual que el iliohipogástrico, se origina del primer nervio lumbar, surge del borde externo del psoas mayor y pasa en sentido oblicuo a través del músculo cuadrado lumbar; a este nivel emerge, un centímetro medial a la cresta iliaca, tiene un trayecto hacia la línea media sobre el oblicuo menor y transverso, en un trayecto paralelo al cordón espermático; proporciona sensibilidad a la piel de las porciones superior e interna del muslo, en varones al pene y escroto, y en mujeres a los labios mayores. ${ }^{7,8}$

\section{CASO CLÍNICO}

Mujer de 42 años sin antecedentes patológicos de importancia, refiere antecedente quirúrgico de plastia inguinal bilateral laparoscópica hace tres semanas. Inició padecimiento actual en el posoperatorio inmediato hace dos semanas con dolor agudo en región inguinal izquierda con irradiación a cara interna de muslo, refiere el inicio inmediatamente después de cirugía, de tipo urente, incapacitante, durante su hospitalización continuó con la sintomatología a pesar de escalar analgésicos, fue egresada al segundo día de posoperada aún con dolor; en su domicilio agregaron medicamentos neuromoduladores, ansiolíticos y antidepresivos sin mejoría alguna, por lo cual acude a consulta por persistir aún el dolor inguinal izquierdo incapacitante que no permitía la deambulación y que provocaba postración en cama desde el primer día del posoperatorio. A la exploración física: dolor con intensidad 10/10 a la palpación superficial en región inguinal izquierda que contrasta con el dolor leve a la palpación 3/10 en región inguinal derecha. Se realizó entonces mapeo de dicha zona por dermatomas para deducir el nervio involucrado; se detectó una clara afectación del territorio del nervio ilioinguinal izquierdo (Figura 1).

Se realizó bloqueo de la región del ilioinguinal con bupivacaína al $0.5 \%$; a los cinco minutos de administrada la anestesia se registró ausencia total de síntomas, debido a ello, se decide su exploración quirúrgica por laparoscopía para encontrar la causa del atrapamiento neural y su posible resolución. La valoración preoperatoria con estudios de laboratorios y prequirúrgicos resultaron normales.

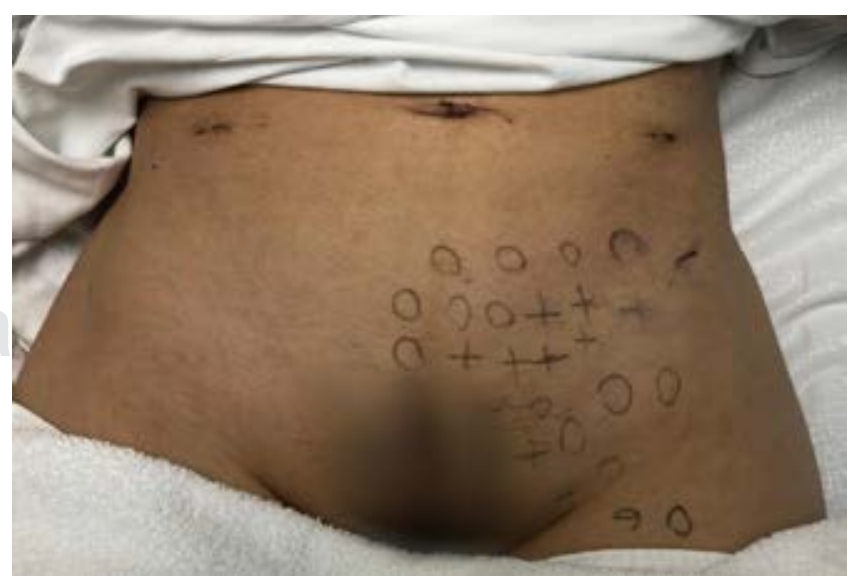

Figura 1: Mapeo por dermatomas con afección de nervio ilioinguinal izquierdo. 


\section{Técnica quirúrgica}

Se realiza laparoscopía en decúbito dorsal con colocación de tres puertos de $11 \mathrm{~mm}$, uno transumbilical y dos en ambos flancos. Se realiza búsqueda intencionada de la emergencia del nervio ilioinguinal a nivel del triángulo del dolor por laparoscopía en la pared abdominal hasta la región inguinal izquierda, el nervio se extiende desde el borde lateral del músculo psoas, y penetra al músculo transverso cerca de la cresta y espina iliaca anterosuperior (Figura 2), encontrando una grapa justo en la emergencia de dicho nervio, se retira grapa (Figura 3) y se realiza además retiro de malla previa (Figura 4) debido a que presentaba migración y no cubría orificio inguinal profundo completamente, se coloca nueva malla Ethicon ${ }^{\circledR}$ Ultrapro y se realiza fijación con pegamento Fix- ${ }^{\circledR}$. La paciente evoluciona de manera satisfactoria sin dolor en territorio de nervio afectado con egreso al tercer día de posoperatorio.

\section{DISCUSIÓN}

Cuando aparece el dolor después del tercer día de posoperatorio de una hernioplastia transabdominal preperitoneal (TAPP) o la hernioplastia extraperitoneal (TEP), algunos autores recomiendan descartar causas morfológicas o complicaciones tempranas de la cirugía (seroma, hematoma) a la exploración o con estudios de radiodiagnóstico. Si ésta no fue la causa, se debe iniciar la línea terapéutica de manejo médico con analgésicos no esteroideos, opioides y neuromoduladores. ${ }^{9}$

El mapeo por dermatomas es una técnica que permite valorar la intensidad del dolor, pero sobre todo el tipo de disestesia presente y los nervios involucrados. ${ }^{10}$ Una vez que el territorio de algún nervio es identificado se procede al bloqueo de los diferentes ramos nerviosos con anestésico local; éste se usa con fines diagnósticos y terapéuticos, pero es transitorio. El dolor persistente e incapacitante en el territorio del nervio comprometido marcará la pauta para la decisión de una intervención quirúrgica. ${ }^{11}$

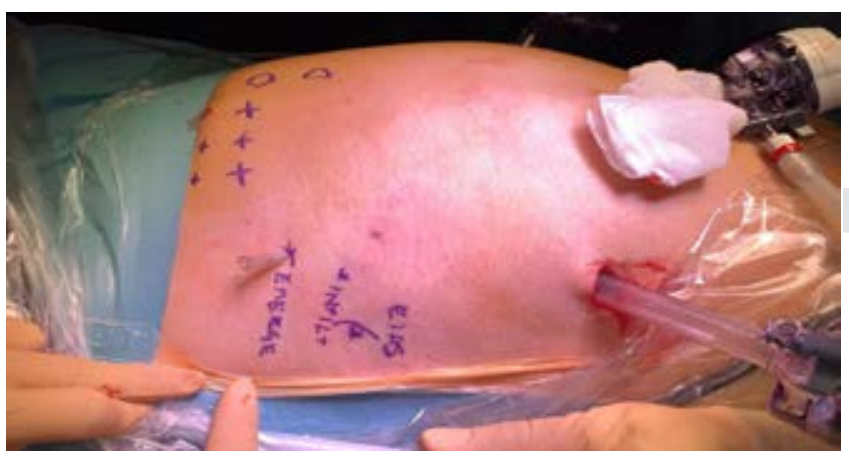

Figura 2: Exploración inguinal por laparoscopía: ubicación de emergencia de nervio ilioinguinal.

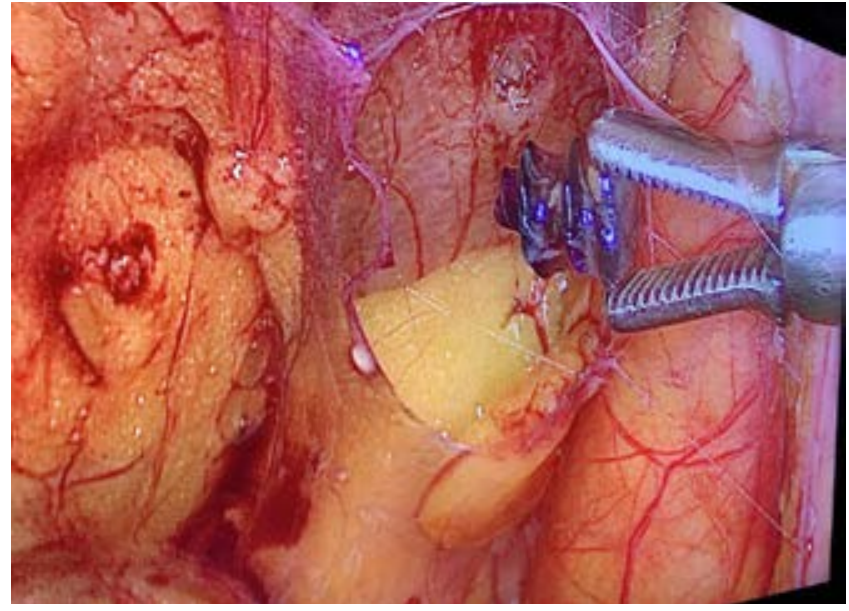

Figura 3: Exploración laparoscópica: retiro de grapa en sitio de nervio ilioinguinal.

La sintomatología del atrapamiento del nervio ilioinguinal se caracteriza por dolor agudo urente $y /$ o pungitivo e hipersensibilidad cutánea en el territorio del nervio atrapado, lo que sugiere su origen en la pared abdominal. El signo de Carnett se identifica como la presencia e incremento de sensibilidad dolorosa en la pared abdominal al efectuar contracción de los músculos abdominales; fue descrito en 1926 y, si se asocia a hiperestesia y alodinia, orienta a determinar el nervio atrapado. ${ }^{12}$

La reparación TAPP de las hernias inguinales se considera preferida por muchos cirujanos. La malla se puede colocar sin fijación con marcada reducción en la incidencia del dolor posoperatorio y dolor crónico por lesiones o atrapamientos de algún nervio, sin aumento significativo en tasas de recurrencia. ${ }^{13}$

Se piensa que estas lesiones nerviosas son secundarias a la colocación de grapas de manera caudal al tracto iliopúbico y lateral, y externos a los vasos iliacos. La fijación de las grapas fuera del ligamento de Cooper penetra en la fascia transversalis y el músculo transverso del abdomen y aumenta el riesgo de lesión a los nervios ilioinguinal e iliohipogástrico. Con esta teoría en mente, el área craneal al tracto iliopúbico y lateral y medial a los vasos epigástricos inferiores se ha denominado un «área segura» para colocar grapas o tacks por algunos autores. ${ }^{14}$ En la cirugía laparoscópica TEP y TAPP de reparación de hernia inguinal y femoral, la fijación de malla sólo se recomienda en defectos mediales grandes (Clasificación M3 Sociedad Europea de Hernias); si se utiliza fijación, se recomienda la técnica atraumática (pegamento de fibrina, cianoacrilato) para minimizar el riesgo de dolor agudo posoperatorio. La fijación de la malla penetrante sólo debe realizarse en áreas anatómicas seguras donde los nervios no están en riesgo (ligamento de Cooper). ${ }^{15}$ 
Dentro de las guías publicadas por The Hernia Surge Group que incluyó cuatro ensayos controlados aleatorios, no se encontraron diferencias significativas en el dolor posoperatorio agudo entre la fijación con pegamento y grapas; sólo una revisión sistemática reveló una incidencia significativamente mayor de dolor cuando el grupo de las grapas se comparó con el grupo del pegamento como medio de fijación. En contraste, tres de los seis ensayos controlados aleatorios y dos de los tres ensayos de casos y controles no informaron diferencias significativas. ${ }^{16}$

Cuando el tratamiento médico para el dolor falla, se pueden utilizar diferentes métodos quirúrgicos; se considera una indicación para la reexploración temprana que el paciente presente dolor inmediatamente después de la cirugía de hernia inguinal (agudo) y es incoercible a las medidas terapéuticas y analgésicas. El tratamiento quirúrgico del dolor después de un abordaje posterior, como la laparoscopía, se puede dividir en dos métodos: el primero es eliminar las grapas y la malla con un método laparoscópico; el segundo es una neurectomía que debe reservarse para manos expertas. ${ }^{17}$

El éxito del abordaje laparoscópico para la remoción de las grapas helicoidales se ve limitado a veces por el hecho de que éstos están profundamente incrustados en los tejidos preperitoneales y no se localizan fácilmente con una simple vista laparoscópica. Wong J y colaboradores reportaron el uso de fluoroscopía para ayudar a identificar las grapas que no pudieron visualizarse por vía laparoscópica. La neuralgia desaparece en la mayor parte de los casos después de la eliminación exitosa de la grapa sin déficit neurológico. ${ }^{18}$

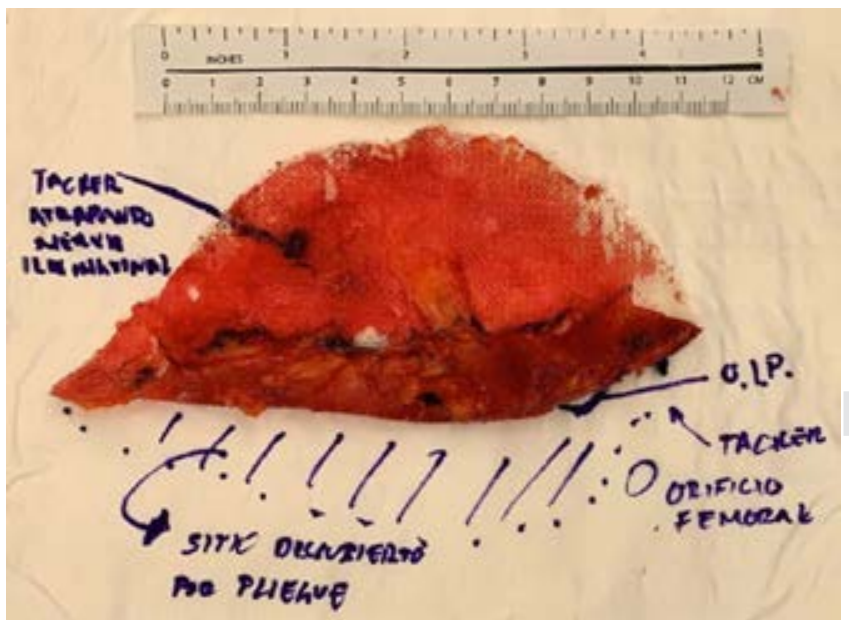

Figura 4: Retiro de malla previa con esquema en relación a su colocación previa.

$\mathrm{OIP}=$ Orificio inguinal profundo .
La extirpación de la malla quirúrgica «meshectomy», ya sea parcial o completa, es un procedimiento quirúrgico que puede considerarse cuando se estima que la malla es el origen del dolor neuropático secundario a una hernioplastia. El retiro de la malla puede ser complejo y en ocasiones una técnica de rescate, ya que la cirugía en el tejido fibrótico puede aumentar considerablemente las posibilidades de complicaciones. Además, la pared posterior o el canal inguinal pueden requerir un refuerzo para evitar la aparición de hernias recurrentes. Esto se puede hacer mediante una nueva inserción de malla o mediante una reparación con tensión. ${ }^{19}$

La primera referencia de una neurectomía laparoscópica fue en 1997 por abordaje retroperitoneal. ${ }^{20}$ La neurólisis o neurectomía selectiva de los nervios ilioinguinal, iliohipogástrico y genitofemoral se realizan con la extirpación de la malla y material de fijación y su revisión; estos dos tratamientos son aceptados para el tratamiento quirúrgico del dolor neuropático refractario a las medidas conservadoras y posiblemente sea la opción más efectiva en casos severos con dolor crónico y lesión nerviosa. En la actualidad, la neurectomía por vía laparoscópica está indicada en los casos con antecedente de cirugía endoscópica o en las neurectomías fallidas por abordaje abierto. ${ }^{21,22}$

\section{CONCLUSIÓN}

El dolor agudo en el posoperatorio inmediato es una entidad que pocas veces requiere de exploración quirúrgica, a menos que sea incapacitante y que no responda a las diferentes líneas de manejo conservador.

El uso de pegamento para la fijación de la malla reduce significativamente el dolor temprano posoperatorio y la inguinodinia con menor riesgo de atrapamiento de los nervios del plexo lumbar.

Es importante identificar a aquellos pacientes con dolor posoperatorio con más de tres días después de la hernioplastia inguinal laparoscópica con sospecha de dolor neuropático; la inyección local de anestésico ofrece información diagnóstica con respecto a la etiología del dolor, si el alivio es transitorio probablemente se beneficien de una reintervención quirúrgica.

No existe suficiente bibliografía sobre el tratamiento adecuado en el dolor agudo incapacitante y de origen neuropático, en particular después de la hernioplastia por TAPP o TEP, así como la exploración inguinal y la nefrectomía desde una perspectiva puramente pragmática.

\section{AGRADECIMIENTOS}

Al American British Cowdray Medical Center Ciudad de México, por proporcionarnos lo necesario para llevar una práctica médica con calidad y calidez. 


\section{REFERENCIAS}

1. Callesen T, Bech K, Nielsen R, Andersen J, Hesselfeldt P, Roikjaer $\mathrm{O}$ et al. Pain after groin hernia repair. $\mathrm{Br}$ J Surg. 1998; 85: 1412-1414.

2. Hidalgo M, Castellón C, Figueroa JM, Eymar JL, Moreno GE. Complicaciones de la cirugía de las hernias. Cir Esp. 2001; 69: 217-223.

3. Arias CL, Vázquez SH, Kleinfinger MS. Reparación de hernia inguinal laparoscópica con uso de materiales autoexpandibles. Rev Mex Cir Endoscop. 2011; 12: 18.

4. Moreno-Egea A. Neurectomía laparoscópic a transabdominal preperitoneal como tratamiento de la inguinodinia. Experiencia personal y detalles de la técnica quirúrgica. Rev Hispanoam Hernia. 2018; 6 (2): 69-74.

5. Rab M, Ebmer And J, Dellon AL. Anatomic variability of the ilioinguinal and genitofemoral nerve: Implications for the treatment of groin pain. Plast Reconstr Surg. 2001; 108: 1618-1623.

6. Reinpold W, Schroeder AD, Schroeder M, Berger C, Rohr $M$, Wehrenberg $U$ et al. Retroperitoneal anatomy of the iliohypogastric, ilioinguinal, genitofemoral, and lateral femoral cutaneous nerve: Consequences for prevention and treatment of chronic inguinodynia. Hernia. 2015; 19: 539-548.

7. Graham DS, MacQueen IT, Chen DC. International inguinal neuroanatomy: implications for prevention of chronic postinguinal hernia pain. Int J Abdom Wall Hernia Surg. 2018; 1: 1-8.

8. Morales-Barrios J, Flores-Rangel GA, Chávez-Villacaña E. Inguinodinia. Rev Mex Anest. 2016; 39: 122-128.

9. Kockerling F, Schug-Pass C. Early Surgical intervention following inguinal hernia repair with severe postoperative pain. Front Surg. 2017; 4: 67.

10. Donati M, Brancato G, Giglio A, Biondi A, Basile F, Donati A. Incidence of pain after inguinal hernia repair in the elderly. A retrospective historical cohort evaluation of 18-years' experience with a mesh \& plug inguinal hernia repair method on about 3000 patients. BMC Surg. 2013; 13: S19.

11. Demirci A, Mercanoglu E, Türker G, Gurbet A, Kaya F, Anil A et al. Bloqueo de los nervios ileohipogástrico/ilioinguinal en corrección de hernia inguinal para el tratamiento del dolor en el posoperatorio: comparación entre la técnica de marcas anatómicas y la guiada por ultrasonido. Rev Bras Anestesiol. 2014; 64: 350-356.

12. Funes RJ, Domínguez GL, Ramme CC, Domínguez CL. Síndrome de atrapamiento del nervio ilioinguinal. Acta Médica Grupo Ángeles. 2018; 16: 233-235.

13. Darwish AA. Tack fixation versus nonfixation of mesh in laparoscopic transabdominal preperitoneal hernia repair. The Egyptian Journal of Surgery. 2016; 35: 327-331.

14. Lantis JC 2nd, Schwaitzberg SD, Tack entrapment of the ilioinguinal nerve during laparoscopic hernia repair. J LaparoendoscAdv Surg Tech A. 1999; 9: 285-289.

15. Wolfgang R. Risk factors of chronic pain after inguinal hernia repair: a systematic review. Innov Surg Sci. 2017; 2 (2): 61-68.

16. The Hernia Surge Group. International guidelines for groin hernia management. Hernia. 2018; 22: 1-165. https://doi. org/10.1007/s'0029-017-1668-x.

17. Lange JF, Kaufmann R, Wijsmuller AR, Pierie JP, Ploeg RJ, Chen DC et al. An international consensus algorithm for management of chronic postoperative inguinal pain. Hernia. 2015; 19 (1): 33-43. doi: 10.1007/sº029-014-1292-y.

18. Wong J, Anvari M. Treatment of inguinodynia after laparoscopic herniorrhaphy: a combined laparoscopic and fluoroscopic approach to the removal of helical tackers. Surg Laparosc Endosc Percutan Tech. 2001; 11 (2): 148151.

19. Zwaans WAR, Koning GG, Gurusamy KS, van Kleef M, Scheltinga MRM, Roumen RMH. Surgical interventions for the management of chronic groin pain after hernia repair (postherniorrhaphy inguinodynia) in adults. Cochrane Database of Systematic Reviews. 2017; 4: CD012630.

20. Mayagoitia GJ, Baca PJ, Cisneros MH, Domínguez CL. Triple neurectomía laparoscópica por dolor inguinal crónico posoperatorio (inguinodinia). Reporte de caso. Rev Mex Cir Endoscop. 2018; 19: 25-29.

21. Chen DC, Hiatt JR. Operative management of refractory neuropathic inguinodynia by a laparoscopic retroperitoneal approach. JAMA Surg. 2013; 148: 962-967. doi: 10.1001/ jamasurg.2013.3189.

22. Moreno-Egeaa A, Borras RE. Neurectomía laparoscópica transabdominal retroperitoneal, selectiva y ambulatoria, para tratar el dolor neuropático inguinal refractario. Sociedad Hispanoamericana de Hernia. 2014; 2: 67-71. 